\title{
Femtosecond measurements of electric fields: from classical amplitudes to quantum fluctuations
}

\author{
Claudius Riek, Denis V Seletskiy and Alfred Leitenstorfer \\ Department of Physics and Center for Applied Photonics, University of Konstanz, \\ D-78457 Konstanz, Germany \\ E-mail: aleitens@uni-konstanz.de
}

\begin{abstract}
Ultrabroadband electro-optic sampling is presented as an extremely sensitive technique to detect electric field amplitudes in free space. The temporal resolution provided by few-femtosecond laser pulses results in a bandwidth exceeding $100 \mathrm{THz}$, potentially covering the entire infrared spectral range. A pedagogic introduction to the operational principle of the method is given along the lines of a classical coherent input field and a zincblende-type electrooptic sensor. We then show that even the bare vacuum fluctuations of the electric field in the quantum ground state may be detected. This time-domain approach to quantum physics operates directly on sub-cycle scales where no local energy conservation holds. Therefore, signals may be obtained from purely virtual photons without amplification to finite intensity.
\end{abstract}

Keywords: time-domain spectroscopy, electromagnetic fields, quantum optics

\section{Introduction}

Despite being an electromagnetic wave, light is typically detected as a time-averaged flux of energy by e.g. measuring the photocurrent that emerges due to generation of electron-hole pairs in a $\mathrm{p}-\mathrm{i}-\mathrm{n}$ diode that absorbs photons. This fact is due to the extremely high frequencies in the terahertz to petahertz $\left(1 \mathrm{THz}=10^{12} \mathrm{~Hz}, 1 \mathrm{PHz}=10^{15} \mathrm{~Hz}\right)$ range. Consequently, direct resolution of the oscillations of the electric field of a light wave requires femto- to attosecond temporal precision ( $1 \mathrm{fs}=10^{-15} \mathrm{~s}, 1$ as $=10^{-18} \mathrm{~s}$ ). Two different routes exist to achieve this goal that both rely on ultrashort laser pulses [1]. A first group of techniques is based on local acceleration of electrons in a spatially confined region which are released within a short time 


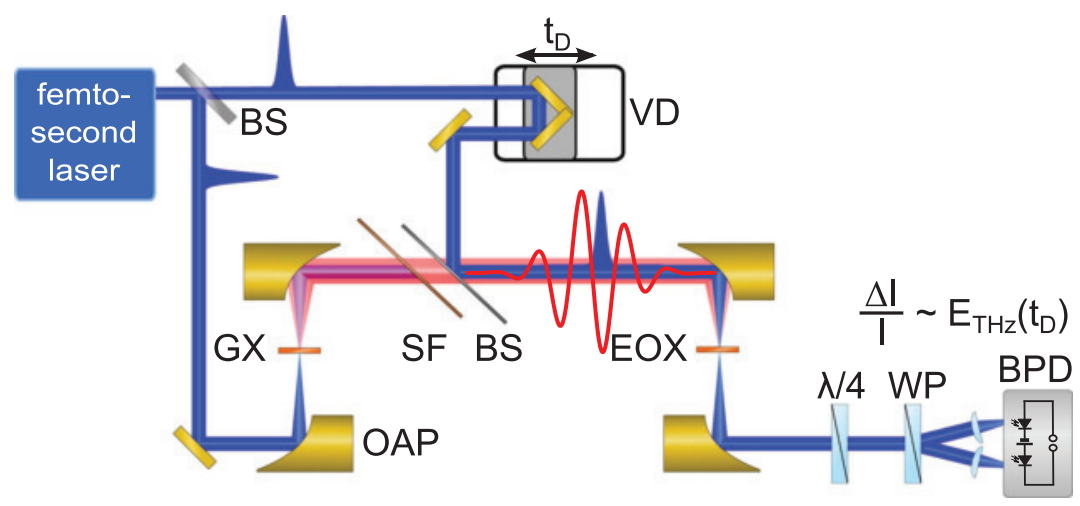

Figure 1. Setup for time-domain spectroscopy based on ultrafast electro-optic sampling. BS: beam splitter; OAP: off-axis parabolic mirror; GX: generation crystal; SF: spectral filter; VD: variable optical delay line; EOX: electro-optic crystal; $\lambda / 4$ : quarter-wave plate; WP: Wollaston polarizer; BPD: balanced pair of photodiodes.

interval by resonant optical excitation. Photoconductive switches are gated by femtosecond generation of electron-hole pairs in a semiconductor material. Their response is typically limited to far-infrared or terahertz frequencies due to the finite photocarrier lifetime $[2,3]$. This bandwidth restriction is relaxed when detecting photocurrents that are proportional to the accumulated time integral of an electric-field waveform. Semiconductor-based photoconductive devices $[4,5]$ or impulsive plasma generation in dense gases [6] then open up the entire infrared region. Attosecond streaking with free electrons from extreme-ultraviolet excitation of atoms in ultrahigh vacuum finally covers visible frequencies [7]. The second set of approaches exploits a nonresonant optical nonlinearity to study light fields with sub-cycle resolution. Especially, the linear electro-optic ('Pockels') effect [8] is attractive for sensitive sampling of electric fields in free space [9-12]. Since only a phase shift of the ultrashort probe pulse is measured and no conservation of energy is required locally on a sub-cycle time scale, this technique even enables access to the quantum fluctuations of the electromagnetic field in its ground state $[13,14]$. In this work, we first develop the fundamentals of ultrafast electrooptic sensing (section 2). We then derive the amplitude of electric-field vacuum fluctuations in free space (section 3) and explain how to detect them directly with this technique (section 4). Throughout this text, the terms 'far-infrared' and 'terahertz' will be used interchangeably to indicate the frequency interval between approximately $100 \mathrm{GHz}$ and $10 \mathrm{THz}$, while 'mid-infrared' or 'multi-terahertz' will address the approximate range from 10 to $150 \mathrm{THz}$.

\section{Free-space electro-optic sampling of classical field amplitudes}

An elementary setup for time-domain spectroscopy based on electro-optic sampling is depicted in figure 1. A femtosecond laser source emits a periodic train of ultrashort pulses (blue) which is divided into a pump and a probe arm at a beam splitter (BS). The pump pulses are focussed onto an emitter element (GX) that uses their large bandwidth to generate phasestable electric field transients at lower, typically infrared frequencies (red). This goal is accomplished either by optical rectification in a nonlinear optical element or acceleration of resonantly excited photocarriers in a bias field. In order to avoid chromatic aberrations, the broadband emission is often collimated and focussed by reflective optical elements such as 


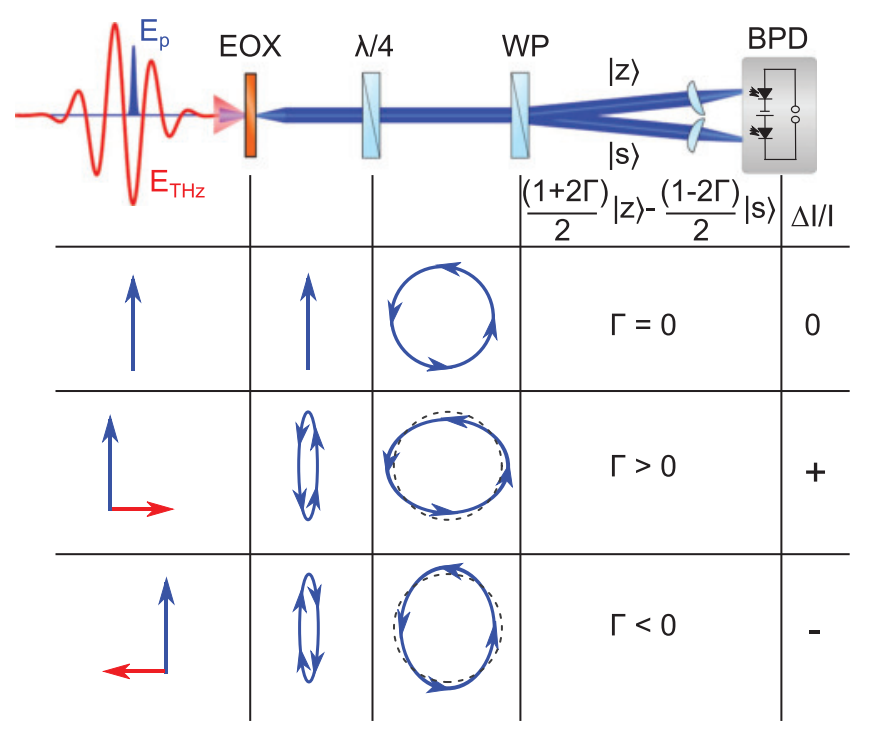

Figure 2. Operational principle of ellipsometric analysis of polarisation state of the probe pulse in electro-optic sampling. $E_{\mathrm{p}}$ : probe pulse envelope (blue); $E_{\mathrm{THz}}$ : signal electric field (red); $\Gamma$ : nonlinear phase shift; $|z\rangle$ and $|s\rangle$ : polarisation components of detected probe field; $\Delta I / I$ : differential photocurrent..

off-axis parabolic mirrors (OAP). A spectral filter (SF) removes residual high-frequency pump light and a second BS superimposes the electric transient with the femtosecond probe pulse. A variable optical delay stage (VD) is used to set the precise timing $t_{D}$ between the infrared waveform and the probe pulse. It consists of a pair of mirrors mounted on a mechanical translation stage that is controlled on a sub-micron scale either with a highprecision stepper motor or a piezoelectric element. The double-pointed arrow indicates the direction of movement. Due to retro-reflection and the finite velocity of light, translation of the VD by $1 \mu \mathrm{m}$ changes $t_{D}$ by $6.67 \mathrm{fs}$. Upon propagation through the electro-optic crystal (EOX), the phase-stable electric transient induces a change in refractive index that is proportional to the local electric field amplitude $E_{\mathrm{THz}}\left(t_{D}\right)$. The influence of the resulting birefringence on the polarisation state of the co-propagating probe pulse is then analysed with an ellipsometer consisting of an achromatic quarter-wave plate $(\lambda / 4)$, Wollaston polarizer (WP) and a pair of balanced photodiodes (BPD). Finally, the infrared waveform is sampled with sub-cycle precision by varying the delay time $t_{D}$.

The operational principle of the ellipsometer is sketched in figure 2 where relevant components of the setup are shown in the top row. The bottom three rows summarise the evolution of the polarisation state of the probe upon its propagation through the apparatus. Before the EOX, both the ultrashort probe wave packet $E_{p}(t)$ (envelope sketched in blue) and the THz field $E_{\mathrm{THz}}\left(t_{D}\right)$ (waveform sketched in red) are linearly polarised along the vertical $|z\rangle$ and horizontal $|s\rangle$ directions, respectively (corresponding directions of polarisation are indicated by blue and red arrows). When $E_{\mathrm{THz}}\left(t_{D}\right)=0$, the polarisation of the probe pulse remains unchanged upon passing through the EOX (second row in figure 2) and it becomes circularly polarised by the $\lambda / 4$. The WP then separates identical power levels into the $|s\rangle$ and $|z\rangle$ polarisation directions which are focussed on one of the pair of BPDs, respectively. Therefore, no differential photocurrent $\Delta I / I$ results. If the electric field $E_{\mathrm{THz}}\left(t_{D}\right)$ has a finite amplitude (third and fourth rows in figure 2), the EOX turns birefringent and the co- 


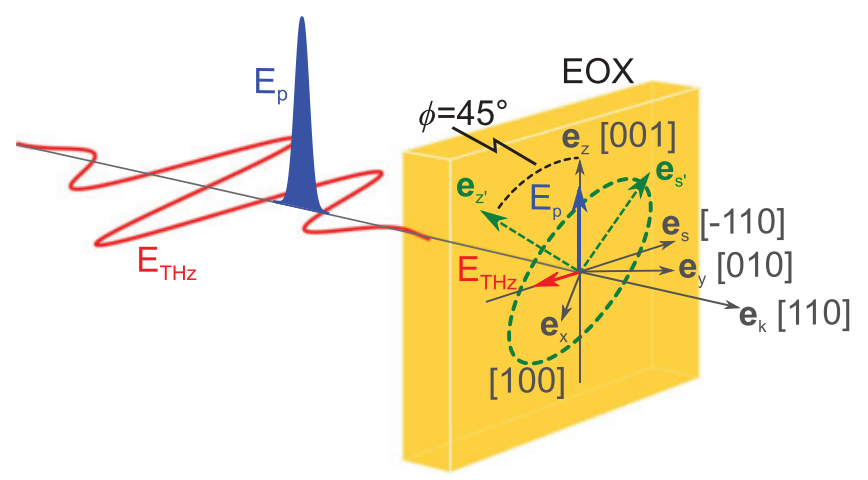

Figure 3. Geometry for exploiting maximum nonlinear phase shift in electro-optic sampling with a detector crystal of zincblende symmetry. The rotated coordinate system in which the refractive index changes are diagonal is indicated by the green dashed arrows and ellipse.

propagating probe pulse experiences a phase shift $\Gamma$. As a result, the output polarisation of the probe deviates from the purely circular state (dashed circle) after the $\lambda / 4$. An imbalance in the power levels of horizontal and vertical detection channels leads to a finite differential photocurrent $\Delta I / I$. For proper alignment of the $\lambda / 4$ (see also next paragraph), the respective photocurrents are proportional to $\Gamma$ where the sign of the phase shift changes with the polarity of $E_{\mathrm{THz}}$, as summarised in the third and fourth rows of figure 2. This fact results in a differential photocurrent signal $\Delta I / I$ which is linearly proportional to the nonlinear phase shift $2 \Gamma$ and hence to the amplitude of the applied field.

To derive the dependence of $\Delta I / I$ on the electric field $E_{\mathrm{THz}}\left(t_{D}\right)$, we consider the geometry in figure 3 which delivers maximum nonlinear phase shifts in free-space electro-optic sampling with a zincblende-type EOX consisting of e.g. ZnTe or GaP. Both $\mathbf{E}_{\mathrm{THz}}$ (red) and the electric field $\mathbf{E}_{p}$ of the probe wave packet (blue envelope) propagate in the [110] crystallographic direction which represents the surface normal of the EOX (identified with unit vector $\mathbf{e}_{k}$ ). Initially, $\mathbf{E}_{p}$ is aligned parallel to the [001] crystallographic direction (unit vector $\mathbf{e}_{z}$ ) and $\mathbf{E}_{\mathrm{THz}}$ is applied along the [-110] axis (unit vector $\mathbf{e}_{s}$ ). A new index ellipsoid (dashed green line in figure 3) then emerges with principal axes $\mathbf{e}_{z}$, and $\mathbf{e}_{s^{\prime}}$ rotated by an angle of $\phi=45^{\circ}$ with respect to $\mathbf{e}_{z}$ and $\mathbf{e}_{s}$ [15]. In the $s-z$ basis, the polarisation of the incoming electric field of the probe $\mathbf{E}_{p, i}$ may be assumed real-valued:

$$
\mathbf{E}_{p, i}=\left(\begin{array}{c}
0 \\
E_{p}
\end{array}\right) .
$$

In the rotated coordinate system $s^{\prime}-z^{\prime}$ of the new index ellipsoid, we obtain

$$
\mathbf{E}_{p, i}^{\prime}=\frac{1}{\sqrt{2}}\left(\begin{array}{cc}
1 & 1 \\
-1 & 1
\end{array}\right) \mathbf{E}_{p, i}=\frac{1}{\sqrt{2}}\left(\begin{array}{l}
E_{p} \\
E_{p}
\end{array}\right) .
$$

The changes of the refractive index induced in $s^{\prime}$ and $z^{\prime}$ directions then amount to

$$
\Delta n_{s^{\prime}}=-\Delta n_{\mathrm{z}^{\prime}}=\frac{1}{2} r_{41} n_{0}^{3} E_{\mathrm{THz}, s} \equiv \Delta n,
$$

respectively, considering the electro-optic coefficient $r_{41}$ and the linear refractive index $n_{0}$ of the detector material [15]. The electro-optic tensor originates from the second-order optical nonlinearity which occurs in non-centrosymmetric materials. It describes a change of the 


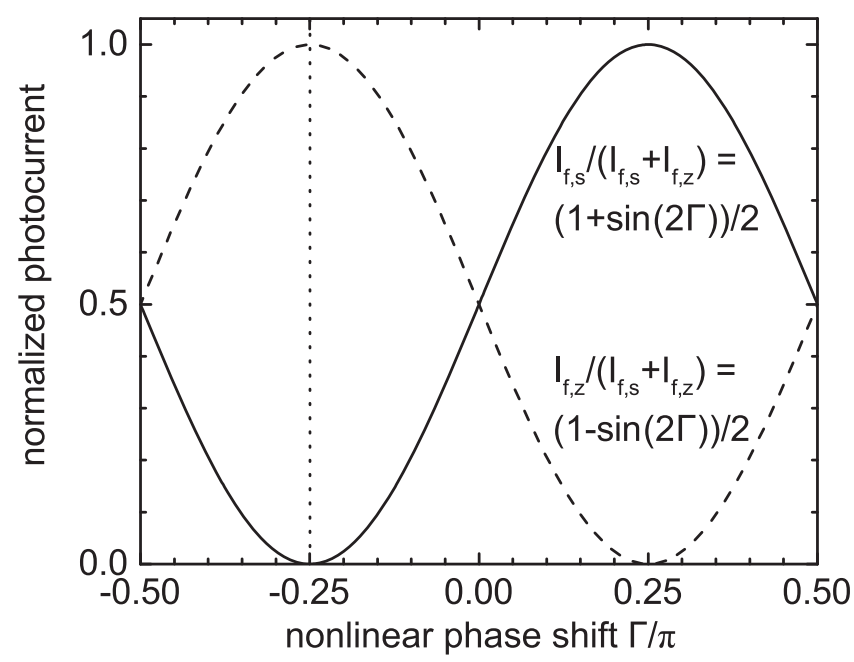

Figure 4. Normalised photocurrents on the two photodiodes forming the optical bridge after the ellipsometer as a function of nonlinear phase shift.

permeability proportional to an applied electric field. $r_{41}$ represents the tensor element which is relevant for the geometry adopted in this paragraph. Typical values of the electro-optic coefficients for the materials and frequency ranges discussed here are in the order of several $10^{-12} \mathrm{~m} \mathrm{~V}^{-1}$. After co-propagation through the electro-optic sensor of thickness $l$, the complex-valued probe electric field $\mathbf{E}_{p \text {,EOX }}^{\prime}$ at the exit surface is

$$
\mathbf{E}_{p, \mathrm{EOX}}^{\prime}=\frac{E_{p}}{\sqrt{2}}\left(\begin{array}{c}
\mathrm{e}^{\mathrm{i} \Gamma} \\
\mathrm{e}^{-\mathrm{i} \Gamma}
\end{array}\right), \text { where } \Gamma=\frac{\omega \Delta n l}{c}
$$

denotes the nonlinear phase shift, $c$ the velocity of light in free space and $\omega$ the centre angular frequency of the probe wave packet [15]. When the quarter-wave plate is aligned such that a $\pi / 2$ additional phase contribution occurs in $s^{\prime}$-direction, the transmitted probe field reads

$$
\mathbf{E}_{p, \lambda / 4}^{\prime}=\left(\begin{array}{cc}
\mathrm{i} & 0 \\
0 & 1
\end{array}\right) \mathbf{E}_{p, \mathrm{EOX}}^{\prime}=\frac{E_{p}}{\sqrt{2}}\left(\begin{array}{c}
\mathrm{i} \mathrm{e}^{\mathrm{i} \Gamma} \\
\mathrm{e}^{-\mathrm{i} \Gamma}
\end{array}\right) .
$$

Rotating back to the original coordinate system $s-z$, we obtain the final probe field

$$
\mathbf{E}_{p, f}=\frac{1}{\sqrt{2}}\left(\begin{array}{cc}
1 & -1 \\
1 & 1
\end{array}\right) \mathbf{E}_{p, \lambda / 4}^{\prime}=\frac{E_{p}}{2}\left(\begin{array}{l}
\mathrm{i} \mathrm{e}^{+\mathrm{i} \Gamma}-\mathrm{e}^{-\mathrm{i} \Gamma} \\
\mathrm{i} \mathrm{e}^{+\mathrm{i} \Gamma}+\mathrm{e}^{-\mathrm{i} \Gamma}
\end{array}\right) .
$$

The Wollaston prism now separates the two polarisation components along $\mathbf{e}_{s}$ and $\mathbf{e}_{z}$. Using equations (3), (4) and (6) while realising that the current $I_{f_{j}}$ ( $j$ stands for the polarisation components $z$ and $s$ ) at each photodiode is proportional to $E_{p, f, j} E_{p, f, j}^{*}$, one derives a relative differential signal

$$
\frac{\Delta I}{I}=\frac{I_{f, \mathrm{~s}}-I_{f, z}}{I_{f, \mathrm{~s}}+I_{f, z}}=\sin (2 \Gamma) \approx 2 \Gamma=\frac{2 \omega \Delta n l}{c}=\frac{\omega l r_{41} n_{0}^{3} E_{\mathrm{TH} z, \mathrm{~s}}}{c},
$$

where the approximation holds in a limit of small nonlinear phase shifts $\Gamma \ll 1$. Note the linear dependence of $\Delta I / I$ on the $s$-component of the electric field $\mathbf{E}_{\mathrm{THz}}$ present in the EOX. To graphically illustrate equations (6) and (7), figure 4 depicts the normalised photocurrent components $I_{f, j} / I$ as a function of nonlinear phase shift $\Gamma$. We see that electronic subtraction 

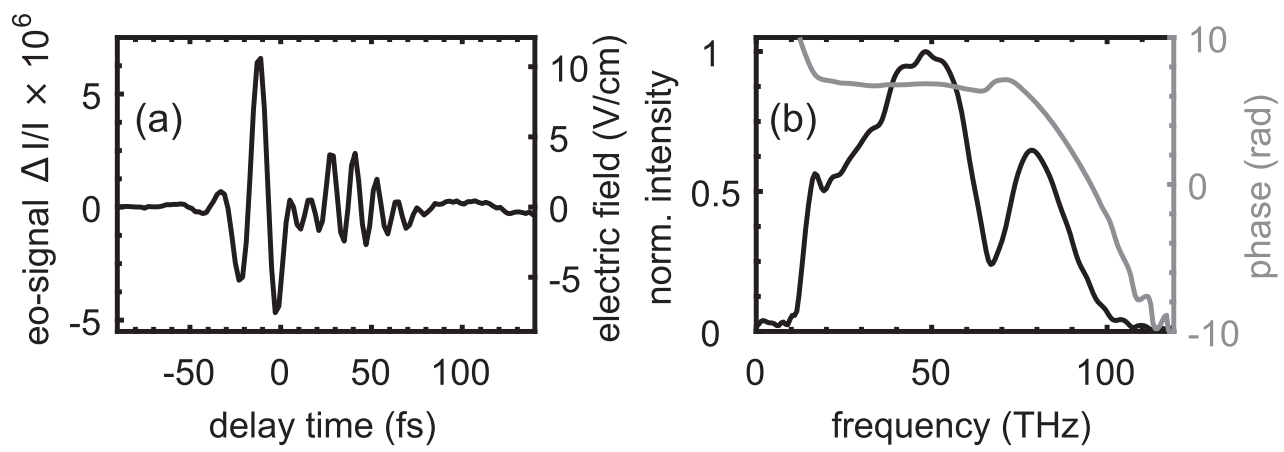

Figure 5. (a) Relative differential photocurrent and deduced electric field amplitude of a coherent transient as a function of delay time. (b) Corresponding amplitude (black) and phase (grey) spectra, as obtained via discrete Fourier transform.

of $I_{f, z}$ (dashed graph) from $I_{f, s}$ (full line) cancels the differential current at $\Gamma=0$. A linear dependence of $\Delta I / I$ on $\Gamma$ emerges locally and technical noise on the probe photocurrent is eliminated. Figure 4 also clarifies the role of the $\lambda / 4$ in the ellipsometer: removal of the quarter-wave 'optical' bias would shift the set-point to $\Gamma=-0.25 \pi$, where the photocurrent components depend quadratically on $\Gamma$ (dotted vertical line in figure 4).

Deriving equation (7), we have assumed that the temporal extent $t_{p}$ of the probe pulse is much shorter than a half-cycle of the field $E_{\mathrm{THz}}$ and its group velocity inside the EOX matches exactly the phase velocity at each detected frequency $\Omega$. In realistic situations, departures from these assumptions together with distortions of the probe envelope due to the dispersion of the EOX and ultimately a retarded temporal response of the electro-optic nonlinearity may be significant. These effects result in a complex-valued and normalised spectral response function $R(\Omega)$ for our electro-optic detection scheme [11, 12, 14]. When sampling electric field amplitudes in a restricted band around a central frequency $\Omega_{c}$ which is far from any electronic or optical phonon resonances of the electro-optic material [13], one may correct equation (7) by an averaged amplitude response $\left|R\left(\Omega_{\mathrm{c}}\right)\right|$. We then have

$$
\frac{\Delta I}{I}=\frac{\omega l r_{41} n_{0}^{3}\left|R\left(\Omega_{c}\right)\right|}{c} E_{\mathrm{THz}, s},
$$

expressing the relative differential photocurrent $\Delta I / I$ in terms of the input electric field amplitude $E_{\mathrm{THz}, s}\left(t_{D}\right)$ in the limit of small nonlinear phase shifts and moderate amplitude and phase distortions.

A mid-infrared electric transient sampled by this scheme is displayed in figure 5. Here, a near-infrared light pulse with $t_{p}=5.8 \mathrm{fs}$ and centre frequency of $\omega / 2 \pi=255 \mathrm{THz}$ was used for probing the electro-optic effect in a $\mathrm{AgGaS}_{2}$ crystal of thickness $l=24 \mu \mathrm{m}$. The crystal structure of this compound is similar to zincblende materials. A small distortion results in uniaxial birefringence that allows phase matching between the signal and probe fields at an angle of $\vartheta=48^{\circ}$. A phase-stable electric-field waveform has been generated by optical rectification of a 7 fs pulse in a $\mathrm{AgGaS}_{2}$ emitter crystal of identical cut and $30 \mu \mathrm{m}$ thickness. The pump pulse was derived from the same modelocked Er:fibre laser system but compressed by a different nonlinear fibre assembly. In this geometry, a small nonlinear dipole emerges only from the deviations from strict zincblende symmetry. Still, our electro-optic detection is readily capable to sample the resulting single-cycle waveform as a function of delay time $t_{D}$ despite its peak electric field of only $10 \mathrm{~V} \mathrm{~cm}^{-1}$ (see figure 5(a)). The amplitude (black 
graph in figure 5(b)) and phase (grey graph) spectra of this transient are easily computed via discrete Fourier transform, demonstrating a frequency content spanning the entire interval between 10 and $100 \mathrm{THz}$. This time-domain method is therefore able to directly measure the full polarisation response of a sample that is put into the beam path. As a convenient example, we take the vibrational absorption of $\mathrm{CO}_{2}$ molecules in ambient air which results in a minimum of the transmitted amplitude spectrum at a frequency of $69 \mathrm{THz}$ and a dispersive feature in the corresponding phase trace (see black and grey lines in figure 5(b), respectively). The polarisation response of the $\mathrm{CO}_{2}$ asymmetric stretch vibrations is also directly seen as a re-emitted field pattern in the time interval between $t_{D}=0 \mathrm{fs}$ and $80 \mathrm{fs}$ of figure $5(\mathrm{a})$, following impulsive excitation by the single-cycle transient. With this technique, one can even investigate the complex-valued susceptibility and its ultrafast temporal evolution in a system that is excited far from thermal equilibrium by yet another ultrashort excitation pulse. A study on the temporal build-up of Coulomb screening in an electron-hole plasma after $10 \mathrm{fs}$ interband excitation of GaAs [16] represents an early example of such a two-time experiment.

\section{The amplitude of electric-field vacuum fluctuations in free space}

The electric field transient in figure 5(a) has been detected exploiting a radio-frequency lockin technique. To this end, the $7 \mathrm{fs}$ pump beam is modulated at half the repetition rate of the femtosecond oscillator of $40 \mathrm{MHz}$ by suppressing every second pulse with a fibre-coupled electro-optic modulator [17]. In this way, we achieve quantum-limited performance in terms of amplitude noise and timing jitter of the probe pulse train, providing a signal-to-noise ratio in the order of $10^{2}$ at a lock-in bandwidth of $2 \mathrm{~Hz}$. This value becomes impressive when one calculates an average content of 0.1 photons in each transient from the temporal shape and field amplitude in figure 5(a), considering a paraxial spot radius of $4 \mu \mathrm{m}$. This value being significantly less than one photon naturally raises the question: what is the fundamental limitation on the measurement accuracy of the electric field?

At this point, we remember that the electric and magnetic field components of light are conjugate observables, resulting in finite fluctuation amplitudes even in the quantum ground state where the intensity vanishes exactly. This fact is elucidated best along the analogy with a one-dimensional mechanical harmonic oscillator of mass $m$, angular frequency $\Omega$ and total energy

$$
H_{\mathrm{HO}}=\frac{1}{2}\left(\frac{p^{2}}{m}+m \Omega^{2} x^{2}\right)=\frac{m}{2}\left(\dot{x}^{2}+\Omega^{2} x^{2}\right) .
$$

It is well known that quantisation of this system results in non-commuting operators for momentum $p$ and displacement $x$. The Gaussian wave function of the ground state extends over a characteristic length $x_{0}=(\hbar / \Omega m)^{1 / 2}$, reflecting the zero-point motion in real space [18]. The total energy of a radiation field in free space with electric and magnetic amplitudes $E=|\mathbf{E}|$ and $B=|\mathbf{B}|$, respectively, and vector potential $A=|\mathbf{A}|$ in the Coulomb gauge is [19]

$$
H_{\mathrm{RF}}=\frac{\varepsilon_{0} V_{0}}{2}\left(E^{2}+c^{2} B^{2}\right)=\frac{\varepsilon_{0} V_{0}}{2}\left(\dot{A}^{2}+c^{2}|\mathbf{k} \times \mathbf{A}|^{2}\right) .
$$

Restricting to one polarisation direction and considering the transverse character of electromagnetic waves, equation (9) maps onto equation (10) by replacing $x \rightarrow A, m \rightarrow \varepsilon_{0} V_{0}$ and $\Omega \rightarrow c k \equiv \Omega$. Instead of $x$ and $p$, an uncertainty product now links $E$ and $B$ or the amplitudes and phases of $E, B$ or $A$, respectively. Again, a Gaussian ground state results with 
an amplitude of vacuum fluctuations connected to a characteristic vector potential $A_{0}=(\hbar /$ $\left.\Omega \varepsilon_{0} V_{0}\right)^{1 / 2}$. In contrast to the mechanical case where calculation of $x_{0}$ is straightforward, it is less clear how to compute a value for $A_{0}$ because outside any cavities, there are no obvious boundaries defining a normalisation volume $V_{0}$. Therefore, we first recall the position operator

$$
\hat{x}=\sqrt{\frac{\hbar}{2 m \Omega}}\left(\hat{a}_{\Omega} \mathrm{e}^{-\mathrm{i} \Omega t}+\hat{a}_{\Omega}^{\dagger} \mathrm{e}^{\mathrm{i} \Omega t}\right)
$$

in a one-dimensional mechanical oscillator. $\hat{a}_{\Omega}$ and $\hat{a}_{\Omega}^{\dagger}$ are the operators for annihilation and creation of a single-mode vibrational excitation with eigenfrequency $\Omega$, respectively. We now have to acknowledge that the free-space radiation field does sustain many eigenmodes which may be expressed by a sum over all possible wave vectors $\mathbf{k}$. In principle, this sum might be replaced by a continuous integral but we will keep it because strictly speaking we measure with a periodic pulse train coming with a well-defined comb of discrete frequencies. Applying the translation rules that lead us from equation (9) to (10) and remembering that $\mathbf{E}=-\dot{\mathbf{A}}$ in the Coulomb gauge, we derive the operator for the free-space field in a plane-wave basis set:

$$
\hat{\mathbf{E}}(\mathbf{x}, t)=\mathrm{i} \sum_{\mathbf{k}, \lambda} \sqrt{\frac{\hbar \Omega}{2 \varepsilon_{0} V_{0}}}\left(\hat{a}_{\mathbf{k}, \lambda} \varepsilon_{\mathbf{k}, \lambda} \mathrm{e}^{\mathrm{i} \mathbf{k x}-\mathrm{i} \Omega t}-\hat{a}_{\mathbf{k}, \lambda}^{\dagger} \varepsilon_{\mathbf{k}, \lambda}^{*} \mathrm{e}^{-\mathrm{i} \mathbf{k x}+\mathrm{i} \Omega t}\right) .
$$

The operators $\hat{a}_{\mathbf{k}, \lambda}$ and $\hat{a}_{\mathbf{k}, \lambda}^{\dagger}$ now annihilate and create a photon with wave vector $\mathbf{k}$, respectively. $\varepsilon_{\mathbf{k}, \lambda}(\lambda=1,2)$ are orthogonal unit polarisation vectors perpendicular to $\mathbf{k}$. We restrict to one polarisation mode, bear in mind the unidirectional character of electro-optic readout and consider the dispersion relation of light in free space where $\Omega=c|\mathbf{k}|$. Since the expectation value $\left\langle\Phi_{0}|\hat{\mathbf{E}}| \Phi_{0}\right\rangle$ of the electric field vanishes in the ground state $\Phi_{0}$, we obtain

$$
\begin{aligned}
\Delta E_{\mathrm{vac}} & =\sqrt{\left\langle\Phi_{0}\left|\hat{\mathbf{E}}^{2}\right| \Phi_{0}\right\rangle}=\sqrt{\left\langle\Phi_{0}\left|\sum_{\Omega=\Omega_{\mathrm{c}}-\Delta \Omega / 2}^{\Omega_{\mathrm{c}}+\Delta \Omega / 2} \frac{-\hbar \Omega}{2 \varepsilon_{0} V_{0}}\left(\hat{a}_{\Omega}-\hat{a}_{\Omega}^{\dagger}\right)^{2}\right| \Phi_{0}\right\rangle} \\
& =\sqrt{\left\langle\Phi_{0}\left|\sum_{\Omega=\Omega_{\mathrm{c}}-\Delta \Omega / 2}^{\Omega_{\mathrm{c}}+\Delta \Omega / 2} \frac{\hbar \Omega}{2 \varepsilon_{0} V_{0}}\left(-\hat{a}_{\Omega}^{2}+\hat{a}_{\Omega} \hat{a}_{\Omega}^{\dagger}+\hat{a}_{\Omega}^{\dagger} \hat{a}_{\Omega}-\hat{a}_{\Omega}^{\dagger}\right)\right| \Phi_{0}\right\rangle} \\
& =\sqrt{\sum_{\Omega=\Omega_{\mathrm{c}}-\Delta \Omega / 2}^{\Omega_{\mathrm{c}}+\Delta \Omega / 2} \frac{\hbar \Omega}{2 \varepsilon_{0} V_{0}}}
\end{aligned}
$$

for the root-mean-square (rms) fluctuation amplitude of the quantum vacuum. $\hat{a}_{\Omega}$ and $\hat{a}_{\Omega}^{\dagger}$ are the operators for annihilation and creation of a photon with angular frequency $\Omega$, respectively. The last step results noting that solely $\hat{a}_{\Omega} \hat{a}_{\Omega}^{\dagger}$ yields a non-vanishing contribution for $\Phi_{0}$ and with the bosonic commutation relation $\left[\hat{a}_{\Omega}, \hat{a}_{\Omega}^{\dagger}\right]=1$. We have responded to the fact that the time-domain measurement is sensitive to a limited frequency range by including only an interval of $\Delta \Omega$ around $\Omega_{c}$. This step is critical to ensure convergence of the sum in equation (13). The lateral extension of the volume $V_{0}$ is now easily identified with the transverse cross section of the high-frequency probe beam inside the EOX. A full analysis based on quantisation of free space in a basis of Laugerre-Gaussian mode functions [14] shows that $A_{\text {eff }}=w_{0}^{2} \pi$, where $w_{0}$ is the paraxial spot radius of the probe. Consequently, we set $V_{0}=L \times w_{0}^{2} \pi$ and only the longitudinal extension $L$ of the probing volume remains to be determined. Since our electro-optic crystal is shorter than the Rayleigh range of the multi- $\mathrm{THz}$ transverse mode probed in our experiment, we can apply periodic boundary conditions to the problem. This step results in a density of free-space modes of $L / c$. Therefore, summing over all longitudinal modes within a bandwidth of $\Delta \Omega$ centred at $\Omega_{c}$ eliminates $L$. Owing to the dielectric screening inside the EOX, $\varepsilon_{0}$ needs to be replaced by $n_{0} \varepsilon_{0}$ and we find 


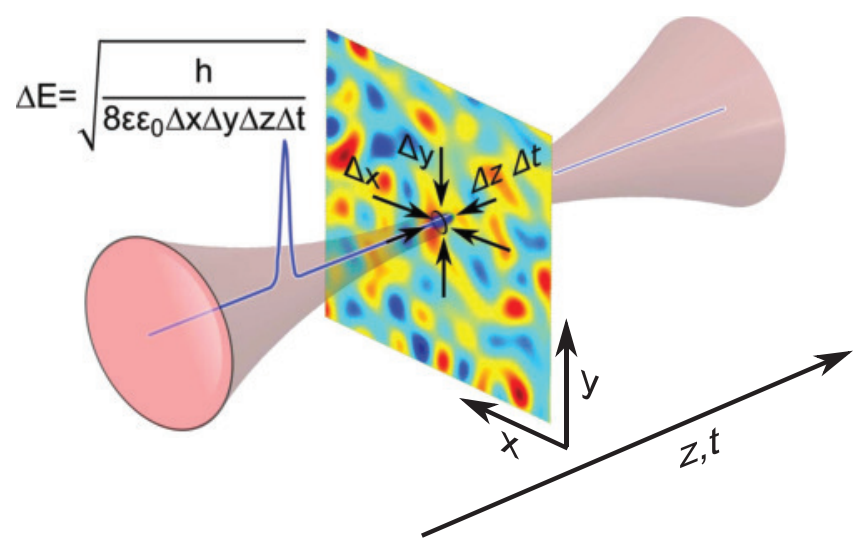

Figure 6. Visualisation of the relevance of the four-dimensional space-time volume in which the co-propagating vacuum electric field is averaged for the quantum amplitude $\Delta E . \Delta x \Delta y$ is determined by the cross section of the transverse mode of the sampling beam (pink) while the spatial length and temporal duration of the probe pulse (blue) sets $\Delta z \Delta t$.

$$
\Delta E_{\mathrm{vac}}=\sqrt{\frac{\hbar \Omega_{c} \Delta \Omega}{(2 \pi)^{2} \mathrm{c} n_{0} \varepsilon_{0} w_{0}^{2}}} .
$$

In our experiment, we had achieved $\Delta \Omega / 2 \pi=66 \mathrm{THz}, \quad \Omega_{c} / 2 \pi=67.5 \mathrm{THz}$, $w_{0}=4.25 \mu \mathrm{m}$ and $n_{0}$ of $\mathrm{AgGaS}_{2}$ is 2.4 , resulting in a vacuum fluctuation amplitude of $\Delta E_{\mathrm{vac}}=20.2 \mathrm{~V} \mathrm{~cm}^{-1}$ [13]. Equation (14) directly shows that the vacuum amplitude is maximised when averaging over a minimal space-time volume. In the electro-optic setup, it is defined by a sampling beam radius of $w_{0}$ and the Fourier transform of the spectral response function $R(\Omega)$ which may be represented by an effective probe pulse duration $t_{p} \approx \pi /$ $\Delta \Omega \approx \pi / \Omega_{c}$. Defining a transverse cross section $\Delta x \Delta y=A_{\text {eff }}=w_{0}^{2} \pi$ and an effective longitudinal length-duration of the probe pulse $\Delta z \Delta t=c \pi^{2} /\left(n_{0} \Omega_{c} \Delta \Omega\right)$, an intuitive form for the rms amplitude of electric-field vacuum fluctuations in a co-propagating reference frame emerges:

$$
\Delta E_{\mathrm{vac}}=\sqrt{\frac{h}{8 \varepsilon \varepsilon_{0} \Delta x \Delta y \Delta z \Delta t}} .
$$

Here the possibility of a reduced speed of light inside a dielectric with refractive index $n_{0}$ and dielectric function $\varepsilon=n_{0}{ }^{2}$ is taken into account. The fact that $n_{0}$ is replaced by $\varepsilon$ proceeding from equation (14) to (15) reflects the modification of only the spatial length $\Delta z$ and not the temporal duration $\Delta t$ of the pulse inside a medium. The physical meaning of equation (15) is sketched in figure 6. A snapshot of positive (red) and negative (blue) amplitudes of the electric vacuum field is shown colour-coded in one plane at a certain instant of time. The smaller is the lateral extension $\Delta x \Delta y$ of the transverse mode of the readout beam (shown in pink), the larger is the range of spatial frequencies that may be detected. Conversely, spatial averaging of the field fluctuations occurs if $\Delta x \Delta y$ gets large, resulting in a decrease of the detected vacuum amplitude. Interestingly, equation (15) is completely symmetric with respect to the longitudinal dimensions where the intensity envelope of the probe enters twice, in the form of both $\Delta z$ and $\Delta t$. This fully four-dimensional character of the vacuum amplitude, sampled in a reference frame moving at the speed of light, reminds us of the fact that relativistic physics underlies electrodynamics and therefore also quantum electrodynamics. 


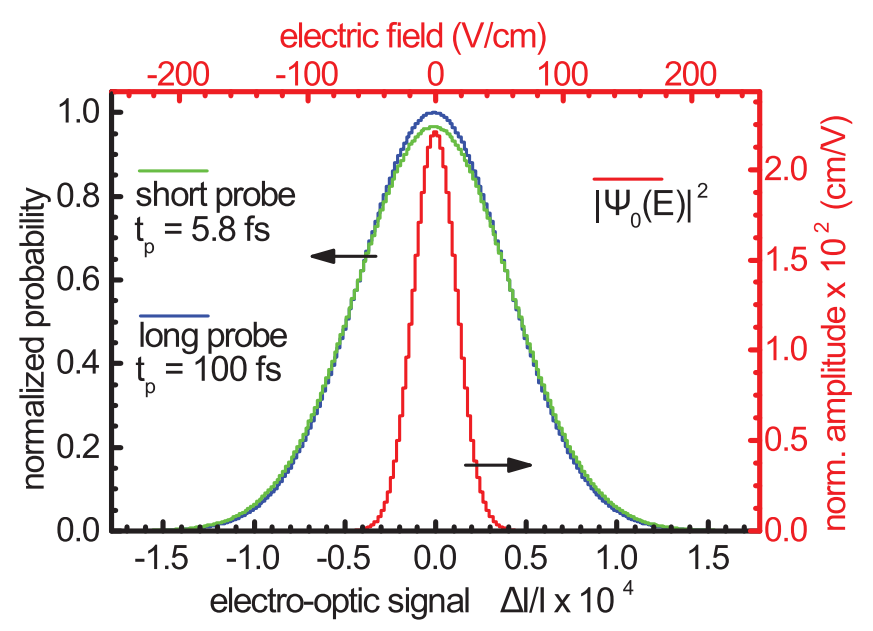

Figure 7. Measurement of the normalised probability distribution of the vacuum electric field as a function of electro-optic signal (bottom axis) or deduced electric amplitude (top axis). The green histogram shows data taken with short pulse duration of $5.8 \mathrm{fs}$ while results for a $100 \mathrm{fs}$ probe pulse are depicted in blue. The red histogram emerges from a deconvolution procedure reconstructing the properties of the electricfield ground state.

\section{Direct sampling of electric-field vacuum fluctuations}

Feasibility of a measurement of the vacuum field amplitude in free space is directly related to the intrinsic noise level of the electro-optic experiment. The coherent near-infrared probe pulses used for readout exhibit a Poissonian photon statistics. In case of a large number of photons per pulse $N_{p}$, this shot noise equals an rms standard deviation of $\Delta N_{p}=N_{p}^{1 / 2}$. With equation (8), the noise-equivalent electric field of electro-optic detection is then defined as

$$
\Delta E_{\mathrm{SN}}=\frac{\Delta N_{p}}{N_{p}} \frac{c}{\omega l r_{41} n_{0}^{3}\left|R\left(\Omega_{c}\right)\right|}=\frac{c}{2 \pi \nu_{p} l r_{41} n_{0}^{3}\left|R\left(\Omega_{c}\right)\right| \sqrt{N_{p}}} .
$$

In our setup, the number of detected probe photons per pulse was $N_{p}=5 \times 10^{8}$ at a carrier frequency of $\nu_{p}=255 \mathrm{THz},\left|R\left(\Omega_{c}\right)\right|=0.41, r_{41}=7.58 \mathrm{pm} \mathrm{V}^{-1}$ and $l=30 \mu \mathrm{m} \mathrm{[13],}$ resulting in $\Delta E_{\mathrm{SN}}=65 \mathrm{~V} \mathrm{~cm}^{-1}$. Since the shot noise of the near infrared probe and the multi$\mathrm{THz}$ vacuum fluctuations are uncorrelated with each other, the two contributions add up statistically. Therefore, the width of the total detected noise distribution may be calculated to increase by a factor of $\left(\Delta E_{\mathrm{SN}}^{2}+\Delta E_{\mathrm{vac}}^{2}\right)^{1 / 2} / \Delta E_{\mathrm{SN}}=1.047$, corresponding to a $4.7 \%$ contribution due to the multi-THz vacuum noise.

To investigate electric-field vacuum fluctuations, we now switch off the coherent input fields that had been characterised in section 2 and search for signals from empty space. Owing to the nonlinear character of the technique, it becomes straightforward to modulate the nontrivial vacuum part of the total noise of the electro-optic detector: according to equations (14) and (15), $\Delta E_{\mathrm{vac}}$ depends on the space-time volume sampled by the probe pulse which may be changed by either manipulating the effective pulse duration $t_{p}$ or the spot radius $w_{0}$. At the same time, $\Delta E_{\mathrm{SN}}$ remains unaffected by a change of $t_{p}$ and $w_{0}$, as manifested in equation (16). We have obtained similar results with both options [13]. For the sake of brevity, in this presentation we restrict ourselves to stretching the longitudinal length-duration 
of the probe pulse. To this end, we chirp the sampling pulse to a duration of $100 \mathrm{fs}$ via translating an SF10 prism in the compressor stage used to control the phase spectrum of the probe. Such prism arrangements are used in ultrafast optics to set the correct phase of a femtosecond pulse for achieving minimum pulse duration [1]. SF10 is a heavy flint glass variant whose optical properties result in negligible group velocity dispersion around a wavelength of $1.2 \mu \mathrm{m}$ when applied in a prism compressor. Varying the position of one prism changes the dispersion of the compressor and therefore the resulting pulse duration. To access the statistics of the quantum vacuum, we extended the bandwidth of the radio-frequency lockin amplifier to $1.6 \mathrm{MHz}$ and sampled the differential photocurrent every $5 \mu \mathrm{s}$. The readout values are then summarised in a histogram showing the probability distribution of the electrooptic signal $p(\Delta I / I)$ or the electric field $p\left(E_{\text {total }}\right)$ which may be obtained via equation (8). A small but distinct reduction in peak counts around $p\left(E_{\text {total }}=0\right)$ is observed when comparing the probability distribution obtained with the $5.8 \mathrm{fs}$ pulse (green histogram in figure 7 ) to the measurement with a stretched pulse (blue histogram). Also, the probabilities in the wings of the distribution including the multi-THz vacuum noise (green) are consistently higher than the corresponding values in the blue histogram. Since both histograms contain the same number of $10^{8}$ readouts, their integral areas coincide. Therefore, a reduction in peak height due to the additional vacuum contribution has to come with an identical increase of the width and the maximum change of normalised probability $\Delta p\left(E_{\text {total }}=0\right)$ of $0.04 \equiv 4 \%$ directly corresponds to the difference of the relative noise amplitude measured with and without multi- $\mathrm{THz}$ vacuum fluctuations. This value is in remarkable agreement with the theoretical result of $4.7 \%$ obtained from equation (14) and (16) above. The red histogram in figure 7 emerges from a deconvolution algorithm searching for the best link between the green and blue probability distributions obtained with and without vacuum noise, respectively. This result directly mirrors the ground-state wave function $\left|\Psi_{0}(E)\right|^{2}$ of the electromagnetic field in the polarisation plane and space-time volume which we probe. Again, the standard deviation of $18 \mathrm{~V} \mathrm{~cm}^{-1}$ extracted for the bare vacuum field agrees well with $\Delta E_{\mathrm{vac}}=20.2 \mathrm{~V} \mathrm{~cm}^{-1}$ calculated from equation (14).

\section{Conclusions}

It is stimulating to discuss some fundamental aspects of this experiment. First we point out important facts underlying our capability to directly monitor the vacuum fluctuations. From the only effective part $\hat{a}_{\Omega} \hat{a}_{\Omega}^{\dagger}$ of the operator extracting the variance of the field in equation (13), we see that our signals come from photons which spontaneously arise and vanish in the fluctuating bare quantum vacuum $\Phi_{0}$. Time-energy uncertainty demands that virtual excitations have a limited lifetime in the order of their oscillation cycle [20]. By copropagating with the electromagnetic field in travelling-wave geometry (see figure 6), our probe 'freezes' time during passage through the thin EOX. Together with the sub-cycle temporal resolution provided by the ultrashort probe wave packet, this fact ensures that we can directly detect signals originating from virtual photons. But does this measurement influence the quantum vacuum at all? Based on the electro-optic change of refractive index $\Delta n \sim r_{41} E_{\mathrm{THz}}$, the co-propagating vacuum field causes a phase shift of the ultrashort probe which is detected. This second-order nonlinear effect operates far from resonance, requires no net transfer of energy and even avoids index modulation at $\Omega / 2 \pi \ll \nu_{p}$. Therefore, we may study the vacuum ground state while imposing negligible influence on it. This statement is supported by the Fourier-domain description of electro-optic sampling [12] where sum and difference frequency mixing operate simultaneously. Consequently, recent experiments with 
broken symmetry between up-and down-conversion for local detection of electric field amplitudes [21, 22] warrant a careful analysis.

Second, we recall the analogy $m \leftrightarrow \varepsilon_{0} V_{0}$ motivated by comparing equations (9) and (10). Our experiment directly illustrates how a volume of space and time obtains an 'inertia-like' character through the aspect of statistical averaging over vacuum fluctuations of a quantum field. Note that the relationship between mass and geometry is at the heart of theoretical efforts to solve cosmological questions like e.g. the nature of dark matter and energy [23]. The general expression for the vacuum electric field amplitude found in equation (15) directly emphasises its dependence on the characteristic extensions $\Delta x, \Delta y, \Delta z$ and $\Delta t$ of a fourdimensional space-time volume over which a physical system or measurement integrates.

For an outlook, note that we have recently generated time-locked patterns of squeezed vacuum noise in the mid infrared and we have detected the deviations from the bare vacuum amplitude as a function of time extending the method outlined in this paper [24]. This timedomain approach to quantum physics also represents the first quantum technology in the multi-terahertz frequency range where many cooperative excitations of condensed matter are located. We can now start to perform real quantum spectroscopy [25] probing e.g. strong electronic correlations with non-classical photon fields.

\section{Acknowledgments}

This work was supported by ERC Advanced Grant \#290876.

\section{References}

[1] see, e.g., Weiner A M 2009 Ultrafast Optics (Hoboken, NJ: Wiley)

[2] Auston D H, Cheung K P and Smith P R 1984 Appl. Phys. Lett. 45 284-6

[3] Fattinger C and Grischkowsky D 1989 Appl. Phys. Lett. 54 490-2

[4] Kono S, Tani M, Gu P and Sakai K 2000 Appl. Phys. Lett. 77 4104-6

[5] Ashida M 2008 Japan. J. Appl. Phys. 47 8221-5

[6] Dai J, Xie X and Zhang X-C 2006 Phys. Rev. Lett. 97103903

[7] Goulielmakis E et al 2004 Science 305 1267-9

[8] Pockels F 1906 Lehrbuch der Kristalloptik (Leipzig: Teubner)

[9] Wu Q and Zhang X-C 1995 Appl. Phys. Lett. 67 3523-5

[10] Nahata A, Weling A S and Heinz T F 1996 Appl. Phys. Lett. 69 2321-3

[11] Leitenstorfer A, Hunsche S, Shah J, Nuss M C and Knox W H 1999 Appl. Phys. Lett. 74 1516-8

[12] Gallot G and Grischkowsky D 1999 J. Opt. Soc. Am. B 16 1204-12

[13] Riek C, Seletskiy D V, Moskalenko A S, Schmidt J F, Krauspe P, Eckart S, Eggert S, Burkard G and Leitenstorfer A 2015 Science 350 420-3

[14] Moskalenko A S, Riek C, Seletskiy D V, Burkard G and Leitenstorfer A 2015 Phys. Rev. Lett. 115 263601

[15] Namba S 1961 J. Opt. Soc. Am. 51 76-9

[16] Huber R, Tauser F, Brodschelm A, Bichler M, Abstreiter G and Leitenstorfer A 2001 Nature 414 286-9

[17] Riek C, Kocher C, Zirak P, Kölbl C, Fimpel P, Leitenstorfer A, Zumbusch A and Brida D 2016 Opt. Lett. $413731-4$

[18] see, e.g., Schwabl F 2007 Quantum Mechanics (Berlin: Springer)

[19] see, e.g., Jackson J D 1999 Classical Electrodynamics (New York: Wiley)

[20] Fürst C, Leitenstorfer A, Laubereau A and Zimmermann R 1997 Phys. Rev. Lett. $783733-6$

[21] Porer M, Menard J-M and Huber R 2014 Opt. Lett. 39 2435-8

[22] Keiber S, Sederberg S, Schwarz A, Trubetskov M, Pervak V, Krausz F and Karpowicz N 2016 Nat. Photon. 10 159-62

[23] see, e.g., Overduin J and Fahr H-J 2001 Naturwissenschaften 88 491-503 
[24] Riek C, Sulzer P, Seeger M, Moskalenko A S, Burkard G, Seletskiy D V and Leitenstorfer A 2017 Subcycle quantum electrodynamics Nature at press (doi:10.1038/nature21024)

[25] Dorfman K E, Schlawin F and Mukamel S 2016 Nonlinear optical signals and spectroscopy with quantum light Rev. Mod. Phys. 88045008 